\title{
Recurrent Herpes Simplex Type 2 Virus (Mollaret) Meningitis
}

Beloo Mirakhur, MD, PhD, and Marc McKenna, MD

Mollaret meningitis is a rare form of meningitis that is recurrent, aseptic, mild, and self-limiting. When initially described by Mollaret, this form of aseptic meningitis had no identifiable infecting agent. ${ }^{1}$ New sophisticated diagnostic tools have now identified Herpes simplex type 2 virus as the most commonly isolated agent. ${ }^{2}$ The case presented in this article is of a patient with a history of frequent bouts of recurrent aseptic meningitis spanning a period of 20 years.

\section{Case Report}

The patient was a 52-year-old woman who presented with malaise, nausea, fever, vomiting, headache, and photosensitivity. Her past medical history was significant for 20 previous episodes of documented aseptic meningitis; all the episodes had required hospitalization. Her past medical history was also significant for arthritis, chronic obstructive pulmonary disease, lupus, obstructive sleep apnea, carpal tunnel syndrome, sciatica, hypertension, diabetes mellitus type II, and transient ischemic attacks. Symptoms from her earlier meningitis episodes lasted 3 to 10 days and subsequently cleared without any residual symptoms. Numerous spinal taps performed on her during her previous hospitalizations for meningitis indicated no known specific infectious cause.

She presented to the emergency department complaining of headache, fever, stiff neck, and photophobia. She had a temperature of $100.0^{\circ} \mathrm{F}$, blood pressure of $138 / 83 \mathrm{~mm} / \mathrm{Hg}$, and a heart rate of 85 beats/min. Her physical examination was remarkable for a stiff neck and a mildly decreased sensorium but no other focal neurological signs. Computed tomography, magnetic resonance imaging, and magnetic resonance angiography of her brain

Submitted, revised, 19 January 2004.

From the Chestnut Hill Hospital, Philadelphia, Pennsylvania. Address correspondence to Marc McKenna, MD, Chestnut Hill Hospital, 8815 Germantown Avenue, $5^{\text {th }}$ Floor, Philadelphia, PA 19118 (E-mail: mckennamw@ chh.org). were unremarkable. Cerebrospinal fluid (CSF) from her spinal tap was colorless with a hazy appearance, glucose was $75 \mathrm{mg} / \mathrm{dL}$, and protein was $116 \mathrm{mg} / \mathrm{dL}$, red blood cell count was $2 / \mathrm{mm}^{3}$ with a white blood cell count of 633 . The differential count of the white blood cells was $74 \%$ lymphocytes, $19 \%$ monocytes, and $6 \%$ neutrophiles. CSF was negative for acid fast bacteria and fungus. No cryptococcal antigen or antibodies were present. The Gram stain of the CSF did not show the presence of organisms but was positive for Herpes simplex type 2 virus by polymerase chain reaction (PCR). Serum C3, C4, and total complement levels were within the normal range. Lyme serology was nonreactive. Complete blood count, electrolytes, serum urea nitrogen, and creatinine were within normal limits. There was a mildly elevated peripheral glucose. The patient was positive for anticardiolipin IgA antibody. DS DNA antibody and those for SS-A and SS-B were absent.

At admission, the patient was initially treated with ceftriaxone for possible bacterial meningitis. When the laboratory tests were positive for Herpes simplex type 2 virus, the antibiotic was changed to intravenous acyclovir. This admission was prolonged because of complications from sleep apnea and chronic obstructive pulmonary disease, necessitating intubation of the patient. On recovery, the patient had no neurological deficits and was discharged from the hospital on a prophylactic dose of acyclovir. Acyclovir was dosed at $800 \mathrm{mg}$ twice daily. Two years after initiation of acyclovir, the patient remains symptom-free and has had no further hospitalizations for recurrence of meningitis.

\section{Discussion}

Various viruses can cause aseptic meningitis. ${ }^{3}$ In the adult population, a variant of recurrent aseptic meningitis has been identified, and this syndrome is known as the Mollaret syndrome. Mollaret described this unique form of recurrent aseptic meningitis in $1944 .{ }^{1}$ The clinical presentation of Mollaret meningitis is recurrent episodes of 
meningismus, headache, and fever, separated by symptom free episodes. Each episode can last from a few days to 3 weeks and generally resolves without any clinical intervention. Transient neurological symptoms may occur in some patients but the symptoms resolve with the resolution of the infection. The episodes of meningitis may not be associated with active herpetic lesions on the skin or mucous membranes. The course of the disease, although protracted, is benign, and does not pose a threat to the patient. In general, the symptoms tend to reoccur over a period of 3 to 5 years, although a case lasting more than 28 years has been reported. ${ }^{4}$ Recurrent attacks of Mollaret meningitis usually resolve after a period of 3 to 5 years. ${ }^{5}$

This patient fulfilled the criteria proposed by Bruyn et $\mathrm{al}^{6}$ for the clinical diagnosis: recurrent attacks separated by symptom-free weeks or months, spontaneous remission of symptoms and signs, recurrent episodes of severe headache, meningismus, and fever. Clinical and pathologic evidence of infectious meningitis were absent. Magnetic resonance imaging, magnetic resonance angiography, computed tomography, and electroencephalographic studies ruled out the possibility of a lupus neuropsychiatric illness. The absence of lupus CNS manifestation was further ruled out by the presence of normal levels of complement C3 and $\mathrm{C} 4$.

Recent data suggest that Herpes Simplex type 2 virus is identified as the most common agent causing Mollaret syndrome. ${ }^{2,7,8}$ After the primary infection, Herpes simplex type 2 virus becomes dormant, most commonly within the sensory neurons of the sacral dorsal root ganglia. It is believed that the retrograde seeding of the CSF by the Herpes simplex type 2 virus results in meningitis. With the advent of new diagnostic procedures, such as the PCR, which is now widely available, it has become easy to identify the presence of Herpes simplex type 2 virus in patients with Mollaret syndrome, suggesting that it is a causative agent of benign recurrent meningitis. ${ }^{9}$ The detection of Herpes viral DNA in the CSF does not require any additional procedures; it is a simple test that can be done on the CSF that has already been collected. Detection of Herpes DNA in the CSF will allow the clinician to use abortive and preventive antiviral therapy.

The major problem in assessing the efficacy of any drug therapy is the nature of the disease, which

\section{Table 1. Characteristics of Mollaret Meningitis}

Recurrent episodes of recurrent meningitis

Episodes separated by symptom free periods

Spontaneous remission of symptoms

Transient neurological symptoms in 50\% of cases

No permanent neurological sequelae

Probable causative agent is Herpes simplex type 2 virus

has spontaneous resolution. The rarity of Mollaret syndrome precludes well-documented clinical trials studying the efficacy of various antiviral drugs. Although acyclovir is a safe, effective, and specific anti-Herpes drug, ${ }^{10,11}$ it has not been shown to definitively alter the natural history of the disease, whereas other therapies, including estrogen, steroids, antihistamine, phenylbutazonum, and colchicine have been unsuccessful in the treatment of Mollaret meningitis. ${ }^{12,13}$ The case we present is an interesting case of Mollaret meningitis, with numerous reoccurrences spanning a period of 20 years. To our knowledge, this is the largest number of recurrent episodes documented to occur in an individual patient. In our patient, it took more than 21 hospitalizations before the diagnosis of Mollaret syndrome was made. Several hospital admissions could not have been prevented in this patient but the earlier diagnosis of this disorder might have avoided extensive diagnostic investigations associated with each admission.

\section{Conclusion}

Mollaret syndrome, although rare, should be considered in all persons with recurrent aseptic meningitis (Table 1). It can be diagnosed by PCR analysis of spinal fluid for the presence of viruses, in particular Herpes simplex type $2 .{ }^{14}$ The diagnosis of this syndrome could prevent various hospital admissions for patients, and short-term attacks could be treated with acyclovir. ${ }^{15}$ The efficacy of long-term antiviral prophylaxis is unknown; it might prove to be of some benefit in decreasing the number of recurrences as it did in our patient, who has remained symptom-free on acyclovir, as of this writing, for the last 2 years.

\section{References}

1. Mollaret P. La méningite endothélio-leukocytaire multi-récurrente bénigne. Rev Neurol (Paris) 1944; 76:57-67.

2. Tedder G, Ashley R, Tyler K, Levin M. Herpes 
simplex virus infection as a cause of benign recurrent lymphocytic meningitis. Ann Intern Med 1994;121: 334-8.

3. Beghi E, Nicolosi A, Kurland L, Mulder D, Hauser W, Shuster L. Encephalitis and aseptic meningitis, Olmsted county, Minnesota, 1950-1981: I. Epidemiology. Ann Neurol 1984;16:283-94.

4. Tyler K, Adler D. Twenty-eight years of benign recurring Mollaret meningitis. Arch Neurol 1983; 40:42-3.

5. Stoppe G, Stark E, Patzold U. Mollaret's meningitis: CSF-immunocytological examinations. J Neurol 1987;234:103-6.

6. Bruyn G, Straathof J, Raymakers G. Mollaret's meningitis: differential diagnosis and diagnostic pitfalls. Neurology 1962;12:745-53.

7. Picard F, Dekaban G, Silva J, Rice G. Mollaret's meningitis associated with herpes simplex 2 infection. Neurology 1993;43:1722-7.

8. Hermans P, Goldstein N, and Wellman W. Mollaret's Meningitis and differential diagnosis of recurrent meningitis. Am J Med 1972;52:128-40.
9. Jackson A. Acute viral infections. Curr Opin Neurol 1995;8:170-4.

10. Whitley R, Lakeman F. Herpes simplex virus infections of the central nervous system: therapeutic and diagnostic considerations. Clin Infect Dis 1995;20: 414-20.

11. Townsend G, Scheld W. Infections of the central nervous system. Adv Intern Med 1996;43:403-47.

12. Stamm A, Livingston $W$, Cobbs C, Dismukes W. Failure of colchicine in the treatment of Mollaret's meningitis. Arch Intern Med 1984;144:2265-6.

13. Limburg M, Feenstra L, Lecluse F, Muller H. Mollaret's meningitis responding to phenylbutazonum. Clin Neurol Neurosurg 1985;87:127-30.

14. Aurelius E, Johansson B, Skoldenberg B, Staland A, Forsgren M. Rapid diagnosis of herpes simplex encephalitis by nested polymerase chain reaction assay of cerebrospinal fluid. Lancet 1991;337:189-92.

15. Bergstrom T, Alestig K. Treatment of primary and recurrent herpes simplex virus 2 induced meningitis with acyclovir. Scand J Infect Dis 1990;22:239-40. 